\title{
SOBRE CRISIS Y CRECIMIENTO DEL DERECHO INTERNACIONAL EN SESENTA AÑOS DE NACIONES UNIDAS*
}

\section{Georg NOLTE**}

\begin{abstract}
RESUMEN: Este artículo hace un recuento del progreso normativo internacional a partir de 1945, prestando especial atención al trabajo de la Comisión de Derecho Internacional. Sus éxitos y fracasos son analizados a la luz de las grandes líneas de las relaciones internacionales. El balance no es unívoco: el desarrollo del derecho internacional es una historia ambivalente de constantes sucesiones de crisis y crecimientos. Y en ciertas constelaciones, crecimiento y crisis son sólo apreciaciones distintas sobre el futuro del derecho internacional y, con ello, de las Naciones Unidas.
\end{abstract}

ABSTRACT: This article analyzes the progress of international regulations since 1945, paying special attention to the International Law Commission's work. Success and failures of the Commission's major projects are studied in light of the guidelines of international relations from the early stages of the Cold War to present day. The balance is not a one-sided uniform process: the development of international law is an ambivalent history of constant crisis and growing. In some constellations, crisis and grow are but different views about the future of international law and thus of the United Nations.

RÉSUMÉ: Cet article fait un bilan du processus normatif international depuis 1945, prêtant une attention toute particulière au travail de la commission du droit international. Succès et échecs des principaux projets de la Commission sont analysés à la lumière des relations internationales dans leurs grandes lignes, de l'époque de Guerre froide à nos jours. Le bilan est contrasté: le développement du droit international est l'histoire ambivalente d'une constante succession de périodes de crises et de croissances. Et dans certains cas croissance et crises sont seulement des appréciations différentes de l'avenir du droit international et par la même de l'ONU.

* Este artículo fue publicado previamente en la revista Vereinte Nationen (VN) 5-2005 con el título: "Zu Wachstum und Krisen des Völkerrechts in sechzig Jahren Vereinte Nationen". El autor agradece a Alejandro Rodiles, candidato a doctor en la Universidad de Munich, por la traducción del original.

** Profesor de derecho público, europeo e internacional, así como director del Instituto de Derecho Internacional de la Facultad de Derecho de la Universidad Ludwig-Maximilians de Munich, Alemania. 
SUMARIO: I. Introducción. II. La etapa temprana de la Guerra Fría. III. De la descolonización al final de la Guerra Fría. IV. De 1990 a la fecha. V. Crisis actual y crisis del derecho internacional. VI. Balance.

\section{INTRODUCCIÓN}

Crisis e intentos de reforma caracterizan a las Naciones Unidas en los sesenta años de su existencia. Actualmente no se trata únicamente de un sistema de seguridad colectiva, sino que representan al derecho internacional en su conjunto. A más tardar, a partir de la guerra de Iraq de 2003, se desató una discusión fundamental sobre el rol del derecho internacional en general y la necesidad de reformar a las Naciones Unidas en particular, que por mucho traspasó las fronteras de los círculos especializados. Preguntas como: ¿Ha muerto el derecho internacional ${ }^{1}$ o ¿Todavía tiene la constitucionalización del derecho internacional una oportunidad $?^{2}$ no sólo fueron planteadas en Alemania. Y no sólo de Estados Unidos de América provienen tesis seminuevas sobre los límites efectivos del derecho internacional ${ }^{3}$ y acerca de how international rules die. ${ }^{4}$

El objetivo de las Naciones Unidas de "preservar a las generaciones venideras del flagelo de la guerra" exige "crear condiciones bajo las cuales puedan mantenerse la justicia y el respeto a las obligaciones emanadas de los tratados y de otras fuentes del derecho internacional". Con ello, el preámbulo muestra que los autores de la carta no se hicieron ilusiones sobre la fuerza propia del derecho internacional, y que la carta vive de requisitos que ella misma no puede garantizar. A pesar de todo, las Naciones Unidas exigen participar en la configuración de las condiciones de las cuales depende el cumplimiento del derecho internacional, y no por último mediante el desarrollo del mismo derecho internacional (artículo 1, 3, y artículo 13, 1a., de la Carta de Naciones Unidas).

1 Frowein, Jochen Abr., "Ist das Völkerrecht tot?", Frankfurter Allgemeine Zeitung (FAZ), 23 de julio de 2003.

2 Habermas, Jürgen, "Hat die Konstitutionalisierung des Völkerrechts noch eine Chance?", en id., Der gespaltene Westen, Francfort del Meno, 2004, pp. 113-193.

3 Kagan, Robert, Macht und Ohnmacht-Amerika und Europa in der neuen Weltordnung, Berlín, 2003, pp. 101 y ss.

4 Glennon, Michael J., "How International Rules Die", Georgetown Law Journal, núm. 93, 2005, pp. 939-991. 
Isaiah Berlin dividió a grandes pensadores y poetas en dos categorías: zorros y erizos. Los últimos ven todo a la luz de una idea central; los zorros, en cambio, perciben distintas verdades al mismo tiempo. ${ }^{5}$ Con ello, Berlin no quiso atribuir una respuesta "correcta" a cualquiera de las dos alternativas metodológicas. Con el fin de encontrar una media prudente entre las perspectivas del zorro y el erizo en la interpretación de la crisis actual del derecho internacional - que también puede ser una mezcla de varias crisis - , una revisión del desarrollo de algunas de sus reglas generales podría ser de gran ayuda. Ello deberá ocurrir prestando especial atención a la Comisión de Derecho Internacional de las Naciones Unidas (CDI), un órgano cuyo trabajo siempre ha estado determinado por el estado respectivo del derecho internacional general.

\section{LA ETAPA TEMPRANA DE LA GUERRA FRÍA}

En el primer Programa de Trabajo de la CDI, de 1948, el secretario general de la ONU no encontraba "una razón que pudiera impedir, en apróximadamente veinte años y como resultado de los trabajos de la Comisión, la codificación de casi todo el derecho internacional". ${ }^{6}$

\section{Crecimiento}

A pesar del inicio de la Guerra Fría, fue posible mantener coincidencias fuera de las contradicciones político-ideológicas. Todavía impulsados por la experiencia reciente de la lucha conjunta contra los crímenes contra la humanidad, la Declaración Universal de los Derechos Humanos y la Convención para la Prevención y la Sanción del Delito de Genocidio sentaron, pese a la confrontación de los grandes bloques, los fundamentos para el gran proyecto de articulación de los derechos humanos. Fue también durante la Guerra Fría, y principalmente bajo los auspicios de la $\mathrm{CDI}$, que los proyectos de codificación sobre el derecho del mar (las cua-

5 Berlin, Isaiah, "The Hedgehog and the Fox", Russian Thinkers, East Rutherford, 1979, pp. 22-81.

6 Survey of International Law in Relation to the Work of Codification of the International Law Commission, UN Doc. A/CN.4/1, 5.11.1948, en Anderson et al. (eds.), The International Law Commission and the Future of International Law, Londres, 1998, anexo 2 . 
tro Convenciones de Ginebra de 1958) ${ }^{7}$ y el derecho de las relaciones diplomáticas y consulares (Convenciones de Viena de 1961 y 1963) concluyeron exitosamente. Por supuesto que bajo las circunstancias dadas, esas ramas del derecho se prestaban especialmente bien para el desarrollo progresivo, pues, además de ser materias ideológicamente neutrales, su base codificable ya se encontraba ampliamente en el derecho internacional consuetudinario.

\section{Limites}

En muchos otros campos, no obstante, la apreciación común y el desarrollo del derecho internacional se toparon rápidamente con sus límites. Ello es particularmente cierto respecto a la concretización de los proclamados principios fundamentales del nuevo orden mundial bajo la carta. La adopción del proyecto de la CDI sobre la Declaración de los Derechos y Deberes de los Estados ${ }^{8}$ de 1949 fue pospuesta sine die por la Asamblea General en $1951,{ }^{9}$ lo que constituyó una clara muestra del inicio de la confrontación entre los grandes bloques. Aunque los Principios de Nuremberg, ${ }^{10}$ también formulados por la CDI, no se enfrentaron a oposición en la Asamblea General, ${ }^{11}$ el intento de su concretización, por medio de los proyectos complementarios sobre un código de delitos contra la paz y la seguridad de la humanidad, ${ }^{12}$ y en torno a la cuestión de una jurisdicción penal internacional, ${ }^{13}$ fracasó, entre otras razones, debido a la división respecto a uno de los problemas centrales del derecho internacional: la definición de "agresión". ${ }^{14}$

7 Al respecto, véase Platzöder, Renate, "Conferences on the Law of the Sea”, Encyclopedia of Public International Law (EPIL), 1992, t. I, pp. 748-755.

8 Yearbook of the International Law Commission (YBILC), 1a. sesión, 1949, p. 286.

9 UN Doc. A/RES/596 (VI), 7.12.1951.

10 "Nürnberg Principles", YBILC, 2a. sesión, 1950, t. II, p. 374.

11 UN Doc. A/RES/488 (V), 12.12.1950.

12 "Offences against International Peace and Security, Final Draft Code", YBILC, 6a. sesión, 1954, t. II, p. 112.

13 ILC, YBILC, 2a. sesión, 1950, t. II, p. 378.

14 UN Doc. A/RES/898 (IV), 14.12.1954; "Definition of Aggression", YBILC, 3a. sesión, 1951, t. II, pp. 131-136. Al respecto, véase Tomuschat, Christian, Das Strafgesetzbuch der Verbrechen gegen den Frieden und die Sicherheit der Menschheit, Europäische Grundrechte-Zeitschrift (EuGRZ), núm. 25, t. 2, 1998, pp. 1-7. 


\section{DE LA DESCOLONIZACIÓN AL FINAL DE LA GUERRA FRÍA}

El desarrollo del derecho internacional recibió entonces nuevos impulsos por parte del proceso de descolonización. Como muestra sintomática, valga mencionar aquí la decisión de la CDI de 1961, consistente en darle una nueva y más ambiciosa dirección a su proyecto sobre el derecho de tratados. Hasta ese momento sólo se había pensado en un documento de naturaleza preponderantemente descriptiva; en 1961 se dio el paso hacia el intento de una codificación comprehensiva. La oportunidad que recibirían todos los nuevos Estados de la organización de participar directamente en los trabajos de codificación también motivó la determinación de la CDI, ${ }^{15}$ y la adopción de la Convención de Viena sobre el Derecho de Tratados de 1969 confirmó su acierto.

\section{Desarrollos contradictorios}

El desarrollo del derecho internacional experimentó impulsos aparentemente contradictorios a raíz de la descolonización. Por un lado, los nuevos Estados subrayaban su recién adquirida soberanía nacional, como en la resolución respecto a la soberanía permanente sobre los recursos naturales de la Asamblea General de $1962 .{ }^{16}$ En el mismo tenor se encuentran las demandas por el pleno respeto y la ampliación de los principios de la proscripción del uso de la fuerza y de la no intervención. Estas tendencias culminaron con la Declaración sobre la Inadmisibilidad de la Intervención en Asuntos Internos de los Estados y Protección de su Independencia y Soberanía de $1965,{ }^{17}$ así como con la Declaración sobre los Principios de Derecho Internacional Referentes a las Relaciones de Amistad y a la Cooperación entre los Estados de Conformidad con la Carta de las Naciones Unidas, de $1970 .{ }^{18}$ Por otro lado, eran principalmente los países en vías de desarrollo quienes propagaban la nueva idea acerca de la herencia común de la humanidad (common heritage of mankind). Tras los primeros pasos en relación con el Tratado Antártico (1959), esta idea se manifestó concretamente en el Tratado sobre los Principios

15 ILC, YBILC, núm. 17, 14a. sesión, 1962, t. II, p. 160.

16 UN Doc. A/RES/1803 (XVII), 14.12.1962.

17 UN Doc. A/RES/2131 (XX), 21.12.1965.

18 UN Doc. A/RES/2625 (XXV), 24.10.1970. 
que deben Regir las Actividades de los Estados en la Exploración y Utilización del Espacio Ultraterrestre, incluidas la Luna y otros cuerpos celestes de 1967, así como en la Convención de las Naciones Unidas sobre el Derecho del Mar de 1982. ${ }^{19}$

Empero, después de una observación más detallada, podemos constatar que estos impulsos no eran realmente contradictorios. Su base común consistía en la demanda de los Estados del denominado Tercer Mundo respecto a un desarrollo autodeterminado. Ello exigía tanto autodeterminación política como la participación en bienes, que eran declarados comunes de la humanidad. Esa demanda encontró una respuesta positiva, aunque limitada, por parte de los bloques en oposición. Por una parte, las llamadas superpotencias tenían que acceder, ya sólo por razones de competencia mutua, a las peticiones del Tercer Mundo; por otra, la crisis de Cuba había marcado un claro límite en la conducción de la Guerra Fría. El shock que trajo consigo la crisis de Cuba provocó un cambió en las estrategias del enfrentamiento, que pasó de ser sistemático a desenvolverse por medio de formas indirectas. De tal suerte, se registraron avances significativos en materia de desarme desde principios de los años sesenta, desde el Tratado sobre la Prohibición de Ensayos Nucleares de 1963, así como las convenciones de no proliferación de 1968 y de armas biológicas de 1972, hasta los tratados SALT y ABM del mismo año. ${ }^{20}$ Pero al mismo tiempo, las superpotencias intervenían en Afganistán, Etiopía, Checoslovaquia, Granada, la República Dominicana y Vietnam; intervenciones que justificaban de diversa manera, pero difícilmente por consideraciones humanitarias. ${ }^{21}$

\section{Crecimiento}

El surgimiento del Tercer Mundo contribuyó significativamente al fortalecimiento y a la concreción del derecho internacional como orden jurídico y axiológico. A partir de ello se lograron acuerdos básicos en materias en las que antes la unidad no era posible. Los derechos humanos ocuparon un lugar muy especial al respecto. No fue casualidad que su

19 Cfr. Wolfrum, Rüdiger, “Common Heritage of Mankind”, EPIL I, 1992, pp. 692-695.

20 Al respecto, véase Bruha, Thomas, "Strategic Arms Limitation Talks (SALT)", EPIL IV, 2000, pp. 699-704.

21 Cfr. Nolte, Georg, Eingreifen auf Einladung, Berlín, 1999, pp. 261-295. 
codificación empezara con la Convención Internacional sobre la Eliminación de Todas las Formas de Discriminación Racial de 1965 y culminara provisionalmente con los dos pactos de 1966. Incluso un área más técnica como el derecho de tratados no permaneció intacta ante el fortalecimiento de los derechos humanos, como lo muestra la introducción del concepto de ius cogens en los artículos 53 y 64 del Tratado de Viena. Poco después, la Corte Internacional de Justicia (CIJ) confirmó esa tendencia por medio del concepto de las obligaciones erga omnes. ${ }^{22}$

Para que el derecho internacional pudiera evolucionar como un orden capaz de alzar sus expectativas a la realización de valores determinados, era necesario que las instituciones internacionales, más allá de estar relegadas a funciones de simple coordinación o puramente técnicas, fueran provistas de facultades administrativas con mayor relevancia política. Ello ocurrió mediante la ampliación de las tareas de mantenimiento de la paz en los conflictos del Congo y Chipre, ${ }^{23}$ en el campo económico con los acuerdos sobre materias primas, ${ }^{24} \mathrm{y}$ continuaría con la creación de la Autoridad Internacional de los Fondos Marinos de la Convención sobre el Derecho del Mar de 1982. En una frase multicitada, Wolfgang Friedmann caracterizó dicha tendencia como un cambio del derecho internacional de la coexistencia al de la cooperación. ${ }^{25}$

\section{Limites}

Pero aún antes de que la rivalidad entre las superpotencias llegara a su fin con la caída de la Unión Soviética, aquella etapa de crecimiento en el desarrollo del derecho de gentes también tuvo que toparse con sus propios límites. Las aspiraciones sobre un "nuevo orden económico mundial" encontraban cada vez mayor resistencia en los países occidentales, ${ }^{26}$ en tanto que el aseguramiento de la paz no lograba cobrar forma

22 CIJ, Barcelona Traction, Light and Power Company, Limited, Decisión del 5.2.1970, ICJ Reports, 1970, p. 3 (32).

23 Al respecto, véase Suy, Erik, "United Nations Peacekeeping Operations", EPIL IV, 2000, pp. 1143-1149.

24 Tomuschat, Christian, "Commodities, International Regulation of Production and Trade", EPIL I, 1992, pp. 686-692.

25 Friedmann, Wolfgang, The Changing Structure of International Law, Londres, 1964 , pp. 60-63.

26 Tomuschat, Christian, "New International Economic Order", EPIL III, 1997, pp. 578-582. 
ante la existencia de guerras de procuración (proxy wars). A la proclamación universal de valores sólo seguían hechos concretos en constelaciones excepcionales como Sudáfrica o Rhodesia, o sea en situaciones en las cuales se podía recurrir a una base antirracista compartida. En general, el paso definitivo de la coexistencia mutua a la cooperación siguió siendo un objetivo anhelado.

El hecho de que el viejo proyecto sobre el desarrollo de una jurisdicción penal internacional no registrara progresos y no fuera retomado por la Asamblea General sino hasta 1990, ${ }^{27}$ probablemente tuvo que ver con el espíritu soberanista y anti-intervencionista de la época. Ante dicha constelación, una auténtica investigación jurídica, incluso de las prácticas abiertamente contrarias a los derechos humanos por parte de Estados soberanos, parecía descartada. En el marco de la CDI, los tímidos y paralizados noventa intentos de establecer principios sobre el derecho de los usos de los cursos de agua internacionales para fines distintos de la navegación, ${ }^{28} \mathrm{o}$ bien, para determinar el contenido de la cláusula de la nación más favorecida, ${ }^{29}$ constituyeron ejemplos sintomáticos sobre la imposibilidad de pasar del objetivo abstractamente compartido de la cooperación a la realización concreta en materias centrales del derecho internacional.

\section{DE 1990 A LA FECHA}

Los contemporáneos no deberían estar tan seguros de sí mismos al caracterizar la propia época. No sabemos todavía si el genocidio en Ruanda, los atentados del 11 de septiembre de 2001, el desenvolvimiento de la Organización Mundial del Comercio (OMC), la irrupción de internet o las primeras manifestaciones de una pandemia serán interpretados en veinte años como indicios de una nueva época. Con relativa seguridad se podrá decir que la "globalización" y el "predominio de Estados Unidos de América" jugará un papel importante en comparación con el periodo anterior; por lo menos ese ha sido el caso en la discusión sobre el futuro del derecho internacional. Pero seguramente se podrá describir la etapa

27 UN Doc. A/RES/45/41, 28.11.1990.

28 Watts, sir Arthur, "Non-navigational Uses of International Watercourses, Introductory Note", en id. (ed.), The International Law Commission 1949-1998, Oxford, 1999 , t. II, pp. 1331-1334.

29 Watts, sir Arthur, "Most-Favoured-Nation Clauses, Introductory Note", en id. (ed.), op. cit., nota anterior, t. III, pp. 1793-1795. 
después de 1990, al igual que las fases políticas anteriores, como una en la que el "proyecto del derecho internacional" primero floreció y después fue frenándose de manera crítica y ambivalente.

\section{Crecimiento}

La señal más clara del inicio de una nueva etapa fue la reacción internacional al ataque iraquí contra Kuwait en 1990. Aquí, por primera vez, se desarrollaron y utilizaron eficazmente los instrumentos más duros de las Naciones Unidas. La resolución 687 del Consejo de Seguridad, que contenía un régimen diferenciado de alto el fuego y control para Iraq, fue jurídicamente tan innovadora como otras resoluciones para la solución de conflictos internos, desde Yugoslavia hasta Haití. ${ }^{30}$ Repentinamente, se mostró todo el potencial del sistema de seguridad colectiva de las Naciones Unidas. En el ámbito del comercio internacional, el régimen de la OMC supuso una ampliación temática y un fortalecimiento institucional del antiguo GATT. Bajo las nuevas condiciones, se impuso la idea de universalizar un orden que anteriormente era considerado por muchos como un sistema esencialmente occidental. ${ }^{31} \mathrm{Y}$ también en otros rubros fue posible concluir proyectos que durante mucho tiempo habían estado bloqueados. Dentro de ellos se encuentra, de manera muy especial, la adopción del Estatuto de Roma de la Corte Penal Internacional (CPI), de tratados en materia de desarme como la Convención sobre la Prohibición del Desarrollo, la Producción, el Almacenamiento y el Empleo de Armas Químicas y sobre su Destrucción, y la Convención de Ottawa sobre la Prohibición del Empleo, Almacenamiento, Producción y Transferencia de Minas Antipersonales y sobre su Destrucción, de una serie de convenciones importantes en materia de protección medioambiental, que se dieron como corolario de la Conferencia de Río de 1992,32 así

30 Frowein, Jochen Abr. y Krisch, Nico, “Artículo 39, párrafos 7-10”, en Simma, Bruno (ed.), The Charter of the United Nations-A Commentary, Oxford, 2002.

$31 C f r$. Bogdandy, Armin von, "Chancen und Gefahren einer Konstitutionalisierung der WTO”, en Hallstein, Walter e Institut für Europäisches Verfassungsrecht (eds.), Die europäische Verfassung im globalen Kontext, Baden-Baden, 2004, pp. 65-87; Petersmann, Ernst-Ulrich, "The WTO Constitution and Human Rights", Journal of International Economic Law (JIEL), núm. 3, t. 2, 2000, pp. 19-25.

32 Beyerlin, Ulrich, "Rio-Konferenz 1992: Beginn einer neuen globalen Umweltrechtsordnung?", Zeitschrift für ausländisches öffentliches Recht und Völkerrecht (ZäoRV), núm. 54, 1994, pp. 124-149. 
como también la conclusión por parte de la CDI, en 2001, de las reglas sobre la responsabilidad del Estado por hechos internacionalmente ilícitos. $^{33}$

\section{Limites}

Todavía durante la etapa temprana de la nueva unidad internacional de los noventa se manifestaron los límites del crecimiento: el genocidio que ocurría en Ruanda ante la presencia de las cámaras de televisión coincidió con un mayor énfasis en los intereses nacionales por parte de la potencia estadounidense. Su presidente emitía justo en ese momento, como reacción ante la por "fallida" declarada intervención en Somalia, una directiva según la cual las tropas estadounidenses sólo participarían en operaciones de mantenimiento de la paz cuando éstas correspondieran a los 'intereses nacionales' de Estados Unidos de América. ${ }^{34}$ Una postura más robusta se evidenció con la reacción ante el incumplimiento de sanciones por parte de Iraq, ${ }^{35}$ o bien, con la negociación de acuerdos de protección medioambiental, como el Protocolo de Kioto. ${ }^{36}$ Pero esta tendencia se encrudecería aún más tras la década de los noventa. A la postre, podemos decir que la negociación del Estatuto de la CPI (1998) representó una importante señal al respecto. Cabe recordar que el gobierno estadounidense participó al principio de manera constructiva, pero, envuelto entre el dinamismo de la conferencia y la resistencia interna, no logró firmar el instrumento como miembro fundador. ${ }^{37}$

Ahora bien, la señal de cambio más clara para la política y el derecho internacionales fue, sin duda, la intervención en Kosovo, en 1999. Aquí,

33 UN Doc. A/RES/56/83, 28.01.2002; Crawford, James, The International Law Commission's Articles on State Responsibility, Cambridge, 2002.

$34 \mathrm{Al}$ respecto, véase Thakur, Ramesh, "UN Peace Operations and U.S. Unilateralism and Multilateralism”, en Malone, David M. y Khong, Yuen Foong (eds.), Unilateralism \& US Foreign Policy, Boulder, 2003, pp. 153-180.

35 Tomuschat, Christian, "Using Force against Iraq?", Die Friedens-Warte, núm. 73, t. 1, 1998, pp. 75-81.

36 Assunçao, Lucas, "Turning its Back to the World? The United States and Climate Change Policy", en Malone, David M. y Khong, Yuen Foong (eds.), op. cit., nota 34, pp. 297-317.

37 Nolte, Georg, "The United States and the International Criminal Court", en Malone, David M. y Khong, Yuen Foong (eds.), op. cit., nota 34, pp. 71-94. 
los Estados de la OTAN, bajo el liderazgo de Estados Unidos de América, violaron una norma central de derecho internacional, la proscripción del uso de la fuerza en nombre de uno de sus valores centrales, la protección de los derechos humanos más elementales. ${ }^{38}$ Con ello se desató una crisis del derecho internacional que se vio incrementada con la reacción estadounidense a los atentados del 11 de septiembre de 2001 y con el ataque a Iraq, en marzo de 2003. Legitimar la intervención en Kosovo mediante el postulado de un nuevo equilibrio entre soberanía y protección de los derechos humanos, ${ }^{39} \mathrm{y}$ justificar las operaciones militares contra el Afganistán de los talibán en 2001 por medio de una novedosa interpretación del derecho a la legítima defensa, que además fue avalada por el Consejo de Seguridad, todavía pudo haber sido jurídicamente sostenible. ${ }^{40}$ Empero, la doctrina de la legítima defensa preventiva (preemptive self-defense), la llamada doctrina Bush, formulada en la Estrategia de Seguridad Nacional de 2002, y la guerra contra Iraq en 2003 fueron consideradas inaceptables por la gran mayoría de los Estados y de la población mundial. ${ }^{41}$ El tratamiento de los detenidos por sospecha de terrorismo en la base estadounidense de Guantánamo, en Cuba, fue una clara señal para la opinión pública mundial de que "la última superpotencia" había puesto seriamente en duda, por lo menos para sí misma, las competencias y los parámetros fundamentales del derecho internacional. ${ }^{42}$

38 Simma, Bruno, "NATO, the UN and the Use of Force: Legal Aspects", European Journal of International Law (EJIL), año 10, núm. 1, 1999, pp. 1-22.

39 Tomuschat, Christian, "Völkerrechtliche Aspekte des Kosovo-Konflikts", Die Friedens-Warte, núm. 74, 1-2-1999, pp. 33-37. Para una interpretación distinta, véase Nolte, Georg, "Kosovo und Konstitutionalisierung: Zur humanitären Intervention der NATO-Staaten”, ZaöRV, núm. 59, 1999, pp. 941-960.

40 Tomuschat, Christian, "Der 11. September 2001 und seine rechtlichen Konsequenzen", EuGRZ, vol. 28, 2001, pp. 535-545.

41 Nolte, Georg, "Vorbeugende Gewaltanwendung und gezielte Tötungen: Der Weg in eine andere Rechtsordnung", en Ambos, Kai y Arnold, Jörg (eds.), Der Irak-Krieg und das Völkerrecht, Berlín, 2004, pp. 303-332. También en FAZ, 10 de enero de 2003.

42 Nolte, Georg, "Guantanamo und Genfer Konventionen: Eine Frage der lex lata oder de lege ferenda?", en Fischer, Horst et al. (eds.), Krisensicherung und Humanitärer Schutz. Crisis Management and Humanitarian Protection. Festschrift für Dieter Fleck, Berlín, 2004, pp. 393-404. 


\section{CRISIS ACTUAL Y CRISIS DEL DERECHO INTERNACIONAL}

Actualmente, el derecho internacional se encuentra en una crisis, aunque otras crisis ya se habían presentado a lo largo de los cincuenta y setenta. Si bien es cierto que hay que tener mucho cuidado al derivar una interpretación de la crisis actual a partir de las anteriores, el desarrollo del derecho internacional general desde 1945 sugiere separar "la" crisis del derecho de gentes con mayor precisión. Así, se podrá diferenciar entre tres crisis: 1. La del principio de la proscripción del uso de la fuerza; 2. La del derecho internacional como orden de Estados; y 3. La de elementos particulares del derecho internacional.

\section{La crisis del principio de la proscripción del uso de la fuerza}

Se trata, a todas luces, de la crisis más evidente. Después de la intervención en Kosovo, la proclamación de la doctrina Bush y la última guerra de Iraq, esa gran conquista de la Carta de las Naciones Unidas parece estar a disposición. Por otro lado, las reacciones ante dichos acontecimientos y sus consecuencias han mostrado que la prohibición del uso unilateral de la fuerza es una norma profundamente arraigada en la conciencia de la opinión pública mundial y de casi todos los Estados. ${ }^{43}$ Una observación más detallada demuestra que, en el caso de Kosovo, los Estados de la OTAN no pretendieron realmente tener una justificación legal para la intervención humanitaria unilateral, así como Estados Unidos de América tampoco quiso, mediante la doctrina Bush, establecer un argumento jurídico válido para todos los Estados. Para los actores, el uso unilateral de la fuerza constituye más bien, y en todo caso, una solución de emergencia ante los problemas internacionales de las "catástrofes humanitarias" y de un "terrorismo catastrófico". ${ }^{4}$

43 Thürer, Daniel, "Irak-Krise: Anstoß zu einem Neuüberdenken der völkerrechtlichen Quellenlehre?", Archiv des Völkerrechts, núm. 41, t. 3, 2003, pp. 320 y ss.

44 El término que figura en la versión alemana es massenmörderischer Terrorismus ("mass-murdering terrorism"). Para la versión española se decidió emplear el término "terrorismo catastrófico", al ser éste utilizado por las Naciones Unidas al referirse al mismo fenómeno. C fr. Informe del secretario general de las Naciones Unidas, Un concepto más amplio de la libertad: desarrollo, seguridad y derechos humanos, Nueva York, Naciones Unidas, 2005, UN Doc. A/59/2005, 21.03.2005. 
Si es cierto que fenómenos como el terrorismo catastrófico y una sensibilidad cada vez mayor hacia las catástrofes humanitarias han contribuido a que Estados Unidos de América y sus aliados recurran a métodos tan inaceptables como el uso unilateral de la fuerza militar, también debe existir, al menos en teoría, la posibilidad de encontrar cauces institucionales que hagan de la acción unilateral algo innecesario. Ciertamente, ésta es una de las metas principales de la pretendida reforma de las Naciones Unidas. ${ }^{45}$ El aún no descartado proyecto de ampliación del Consejo de Seguridad obedece a una lógica complicada: el incremento de la eficiencia por medio de la ampliación del número de miembros parece, a primera vista, paradójico. Empero, si con ello se complicase fácticamente el ejercicio del derecho de veto, y la legitimidad derivada de la ampliación facilitase la toma de las medidas más apropiadas para enfrentar los nuevos desafíos, entonces esa aparente paradoja tendría mucho sentido. Todavía es incierto si esta lógica realmente triunfará, y en el intento se debe procurar no restarle valor a la estructura actual del Consejo de Seguridad. Incluso una ampliación de seis nuevos miembros permanentes - con o sin derecho de veto-, no alteraría el principio fundamental del Consejo de Seguridad, i. e. que las potencias más importantes han asumido la responsabilidad de asegurar la paz mundial. Si, a fin de cuentas, nos tuviésemos que conformar con un gremio de 15 miembros, de los cuales cinco contaran con el derecho de veto, seguiríamos identificando, pese a todos los cambios ocurridos desde 1945, a los mismos cinco como las potencias con mayor influencia a nivel mundial. Y poco importa que algunos de ellos no se encuentren dentro de las primeras potencias conforme a determinados criterios cuantificables, como el demográfico. Ahora bien, reconocer esta realidad no significa dejar de exigirles a los cinco permanentes que asimilen su responsabilidad con mayor compromiso y lealtad hacia el interés general, e insistir en la limitación del uso del veto, incluso en su desaparición a largo plazo.

Hay que aceptar que si una revisión de la carta no logra, al menos, amortiguar la crisis por la que atraviesa el principio de la proscripción del uso de la fuerza, tendremos que aprender a vivir con esa nueva constelación. No obstante, las experiencias recientes que han traído consigo las consecuencias del uso unilateral y no autorizado de la fuerza probablemente contribuirán a la revaloración de los principios fundamentales 
y de los procedimientos de la carta. Hay ciertos indicios de que tras las apreciaciones erróneas y las consecuentes decepciones, se empieza a imponer nuevamente un entendimiento, según el cual las amenazas a la paz en virtud del terrorismo y las armas de destrucción masiva no podrán enfrentarse con medidas militares puntuales, sino básicamente por medio de una cooperación estructurada. Mucho dependerá este proceso de la apreciación del gobierno estadounidense y de la opinión pública de ese país, sobre la cual hay que tratar de ejercer influencia.

\section{La crisis del derecho internacional como orden de Estados}

La crisis de la proscripción del uso de la fuerza se yuxtapone con la crisis general del derecho internacional como orden de Estados. En este contexto, la pregunta acerca del uso de la fuerza desempeña un papel fundamental, pues también se trata de la posibilidad de instrumentar un derecho de gentes que ha adquirido, en términos generales, mayor importancia. ${ }^{46}$ Las reacciones colectivas a muchas de las amenazas a la paz desde 1990 han sido interpretadas, especialmente en Alemania, no como simple aplicación de las reglas de la carta, sino como síntomas de la evolución de ésta hacia una auténtica constitución de la comunidad internacional. ${ }^{47}$ Por supuesto que el fenómeno de la 'globalización' ha nutrido esta lectura, toda vez que este concepto no sólo se refiere a la intensificación de la cooperación internacional sino también a la identificación de "nuevos actores", quienes ejercen una influencia nunca antes vista sobre los Estados. Para la opinión pública, las pruebas más claras de ello han sido las conferencias mundiales de los noventa, así como las exitosas negociaciones sobre la Convención de Ottawa y el Estatuto de la CPI. La observación de que el incremento en el movimiento de personas, bienes, capital e información ha disminuido significativamente la capacidad de influencia de los Estados, ha motivado a muchos analistas a interpretar las relaciones internacionales ya no como algo primeramente marcado

46 Ibidem, párrs. 129-132.

47 Frowein, Jochen Abr., "Konstitutionalisierung des Völkerrechts", Berichte der deutschen Gesellschaft für Völkerrecht, 2000, pp. 427-447; Fassbender, Bardo, "The United Nations Charter as Constitution of the International Community", Columbia Journal of Transnational Law, núm. 36, 1998, pp. 529-619. Para una crítica al respecto, véase Orford, Anne, "The Destiny of International Law", Leiden Journal of International Law (LJIL), núm. 17, t. 3, 2004, pp. 441-476. 
por los vínculos interestatales, sino como el desbordamiento de una "comunidad internacional" integrada por los más diversos sujetos de derecho; ${ }^{48}$ algunos incluso llegaron a hablar de una "bukovina global". ${ }^{49}$

Si bien es cierto que la constitucionalización del derecho internacional y la globalización a muchos parecieron dos aspectos de la misma evolución hacia una comunidad internacional bien organizada, también lo es que, a más tardar con el advenimiento del siglo XXI, los lados obscuros de la globalización se mostraron como las causas de una lectura menos optimista de las crisis del derecho internacional. Las "limpiezas étnicas" (Kosovo), el terrorismo (11 de septiembre), el peligro de la proliferación de armas de destrucción masiva (Iraq), así como las posibles soluciones a estos problemas, dieron lugar a tan diversos juicios de valor en la política internacional, que, para algunos, los procesos de toma de decisión por parte de las instituciones competentes, especialmente las Naciones Unidas, ya no eran suficientes. Antes que cualquier otro, Estados Unidos de América decidió hacer frente a esta situación valiéndose de sus propios medios. Por su parte, la conferencia ministerial de la OMC, celebrada en Seattle en 1999, ${ }^{50}$ también significó una desaceleración considerable del desarrollo del derecho comercial internacional. Para ese entonces, ya no se hablaba de la pérdida de importancia del Estado como signo característico de la época, sino más bien del poderío absoluto de un Estado: Estados Unidos de América. ${ }^{51} \mathrm{Al}$ derecho internacional se le planteaba entonces el interrogante de si la hegemonía esta-

48 Cfr. Kwakwa, Edward, "The International Community, International Law, and the United States. Three in One, Two against One, or One and the Same?", en Byers, Michael y Nolte, Georg (eds.), United States Hegemony and the Foundations of International Law, Cambridge, 2003, p. 27. Para un panorama general, véase Paulus, Andreas, Die Internationale Gemeinschaft, Munich, 2001.

49 Teubner, Gunther, "'Global Bukowina'. Legal Pluralism in the World Society”, en Teubner, Gunther (ed.), Global Law without a State, Aldershot, 1997, pp. 3-28.

50 Berrisch, Georg Matthias, "Cancún-was nun?", Recht der internationalen Wirtschaft, núm. 50, t. 1, 2004, pp. 69-75; Wilkinson, Rorden, "The WTO in Crisis", Journal of World Trade, núm. 35, t. 3, 2001, pp. 397-419.

51 Vagts, Detlev F., "Hegemonic International Law", American Journal of International Law (AJIL), núm. 95, t. 4, 2001, pp. 843-848; Byers, Michael y Nolte, Georg (eds.), op. cit., nota 48; Foot, Rosemary et al. (eds.), US Hegemony and International Organizations, Oxford, 2003; Krisch, Nico, Amerikanische Hegemonie und liberale Revolution im Völkerrecht, Der Staat, 43, 2-2004, pp. 267-297. 
dounidense le impregnaría un carácter "imperial". ${ }^{52}$ Para quienes afirmaban el nuevo carácter imperial del derecho de gentes, las señales eran claras. El principio de la igualdad soberana de los Estados habría sido puesto en tela de juicio mediante las demandas del hegemón de exentarse de la aplicación de ciertas reglas cuando a sus intereses - por ejemplo, en la lucha contra el terrorismo- conviniere. Asimismo, la normatividad destinada a garantizar, en lo general y en lo particular, la influencia del hegemón, habría sido reforzada. Esa normatividad se caracterizaría por el uso de conceptos abiertos, cuyo contenido sería determinado por el propio hegemón en el caso concreto, o bien, por la bilateralización de problemas colectivos con el fin de proceder según la máxima divide et impera. $^{53}$

Ante el desplazamiento de la constitucionalización del derecho de gentes por un derecho internacional hegemónico, no debe sorprender que recientemente se le haya prestado tanta atención a la tesis del derecho internacional como derecho administrativo global. En ella se parte de la observación de que la cooperación internacional sigue intensificándose a pesar de las discrepancias más agudas en asuntos de política de alto nivel, es decir, en los temas de "derecho constitucional mundial". Según dicha tesis, ello ocurre no sólo formalmente en el marco de las organizaciones internacionales tradicionales, sino también informalmente, a través de las llamadas redes gubernamentales (government networks), que operan conforme a una coordinación política dificílmente descifrable para la opinión pública. O bien, por medio de la creación de las denominadas coalitions of the willing, que intentan alcanzar sus objetivos fuera de todo foro universal e inclusive formal. ${ }^{54}$ Aquí, la membresía ya no estaría restringida a los Estados, y nuevas reglas, de eficacia internacional, se crearían sin participación estatal alguna. Este tipo de desarrollos son ambivalentes para "el" derecho internacional, pues si bien por un lado puede ganar eficacia, por el otro pone en riesgo la legitimidad y la transparencia en el procedimiento.

52 Krisch, Nico, "International Law in Times of Hegemony", EJIL, núm. 16, t. 3, 2005, pp. 369-408.

53 Krisch, Nico, "Weak as Constraint, Strong as Tool: The Place of International Law in U.S. Foreign Policy”, en Malone y Khong (eds.), op. cit., nota 34, pp. 41-70.

54 Kingsbury, Benedict et al., "The Emerging Global Administrative Law", Law \& Contemporary Problems, núm. 68, t. 15, 2005; Slaughter, Anne-Marie, A New World Order, Princeton, 2004. 


\section{3. ¿Fragmentación o concentración del derecho internacional?}

Aparte de los debates públicos sobre la proscripción del uso de la fuerza, globalización y hegemonía estadounidense, existen otras crisis del derecho de gentes, cuya discusión se realiza principalmente en los círculos especializados. Entre ellas destacan dos cuestionamientos que, a primera vista, arrojan preocupaciones contrastantes sobre el derecho internacional: a) El peligro de su fragmentación; y b) El riesgo de su excesiva concentración. En otras áreas, c) El panorama es mixto.

\section{A. Fragmentación}

Desde 2000, la CDI se ocupa del tema "fragmentación del derecho internacional". ${ }^{55}$ La preocupación que subyace al tema consiste en que, ante la ausencia de un sistema claro de jerarquía normativa en el derecho internacional, la proliferación de regímenes multilaterales con los más distintos objetivos y la más diversa composición, aumenta las probabilidades de que distintas instancias jurídicas interpreten el derecho aplicable de manera contradictoria. Esta posibilidad, que había sido detectada hace tiempo a nivel teórico, cobró importancia práctica en el transcurso de los noventa. Ampliamente conocido es el caso Tadic, en el cual el Tribunal Penal Internacional para la ex Yugoslavia se negó a aplicar el parámetro desarrollado por la CIJ en el caso Nicaragua respecto a la atribución de responsabilidad de los Estados por actos violentos de grupos privados (milicias). ${ }^{56}$ Otros ejemplos atañen la relación del derecho de la OMC con regímenes convencionales en materia de derecho ecológico, laboral y de derechos humanos, o bien, la relación entre los sistemas universal y regionales de protección de los derechos humanos respecto al efecto de las reservas indebidas. ${ }^{57} \mathrm{Y}$ no sólo existen diferencias sobre cómo resolver, tanto material como procesalmente, las incertidumbres respecto a los diferentes sistemas de derecho internacional. La verdad es

55 Report of the International Law Commission, 52a. sesión, 2000, UN Doc. A/55/10, Annex, 1.1.2000; véase en el anexo el estudio del profesor Hafner, Gerhard, Los riesgos resultantes de la fragmentación del derecho internacional.

56 The Prosecutor vs. Dusko Tadic, Judgement, caso núm. IT-94-1-A, 15.7.1999, párrs. 4 y ss.; en http://www.un.org/icty/tadic/appeal/judgement/index.htm.

57 Para un panorama general, véase Koskenniemi, Martti y Leino, Päivi, "Fragmentation of International Law? Postmodern Anxieties", LJIL, núm. 15, t. 3, 2002, pp. 553-579. 
que ni siquiera hay acuerdo en torno a la pregunta inicial, es decir, si la llamada fragmentación realmente es indeseable o si debería acogerse como expresión de un crecimiento, hasta cierto punto natural, y de un pluralismo contemporáneo, propios del orden jurídico internacional. ${ }^{58}$

\section{B. Concentración}

Paralelamente al debate sobre la fragmentación, se discute acerca del rol del Consejo de Seguridad como legislador mundial..$^{59}$ El detonante de esta discusión fue la resolución 1373, adoptada tras los atentados del 11 de septiembre, mediante la cual el Consejo de Seguridad ordenó de manera vinculante a todos los Estados que implementasen en su legislación nacional determinadas disposiciones contenidas en tratados de combate a la financiación del terrorismo, sin considerar que, hasta ese momento, muchos Estados aún no los habían ratificado. ${ }^{60}$ Desde entonces existe la posibilidad de utilizar al Consejo de Seguridad para reducir radicalmente los tiempos y el número de participantes que requiere el proceso normativo internacional tradicional, generalmente mediante la adopción de tratados. Ello se hace con la esperanza de alcanzar, a la mayor brevedad posible, resultados efectivos y de obligatoriedad general. Así, se llegó a discutir la posibilidad de que el Consejo de Seguridad hiciera a un lado, en virtud de una decisión y por tiempo indefinido, la aplicación de la Convención sobre el Derecho del Mar, con el fin de facultar a ciertos Estados a detener e inspeccionar embarcaciones sospechosas de traficar con armas de destrucción masiva. ${ }^{61}$ Contra dichas funciones del Consejo de Seguridad existen, sin duda, objeciones de mucho peso. Todas ellas se basan en la afirmación de que la carta, en su concepción fundamental, no

58 Ibidem, pp. 574-579; Hafner, Gerhard, "Pros and Cons Ensuing from Fragmentation of International Law", Michigan Journal of International Law (MJIL), núm. 25, 2004, pp. 849-83; Fischer-Lescano, Andreas y Teubner, Gunther, "Regime Collisions. The Vain Search for Legal Unity in the Fragmentation of Global Law", MJIL, núm. 25, 2004, pp. 999-1046.

59 Zimmermann, Andreas y Elberling, Björn, Grenzen der Legislativbefugnisse des Sicherheitsrats, VN, 3/2004, pp. 71-77; Nolte, Georg, "Lawmaking through the Security Council", en Wolfrum, Rüdiger y Röben, Volker (eds.), Developments of International Law in Treaty Making, Berlín, 2005, pp. 237-243.

60 UN Doc. S/RES/1373, 28.9.2001.

61 Byers, Michael, "Policing the High Seas", AJIL, núm. 98, t. 3, 2004, pp. 526-545. 
legitima tal función legislativa. ${ }^{62}$ No obstante, el reconocimiento generalizado y reiterado de esa práctica del Consejo de Seguridad por parte de los Estados miembros cuestiona, a su vez, las objeciones mencionadas. Ante la incertidumbre, es importante recordar que esa práctica se circunscribe a la lucha internacional contra el terrorismo y a los intentos por evitar la proliferación de las armas de destrucción masiva, es decir, a materias que según su propia naturaleza no pueden reducirse a campos o situaciones determinados, y en las cuales la referencia al riesgo del uso organizado de la fuerza tiene que ser claramente identificable. ${ }^{63}$ En todo caso, el espíritu de un Leviatán jurídico, opuesto a la producción pluralista tradicional del derecho de gentes, ha emergido. ${ }^{64}$

Ahora bien, si observamos con mayor detenimiento los fenómenos de la "fragmentación" y la "concentración" —incluyendo la jerarquización-, podemos constatar que esos desarrollos del derecho internacional no son tan contradictorios como aparentan. La fragmentación también puede ser contemplada como consecuencia del proceso evolutivo de algunos elementos particulares del orden jurídico internacional. Este proceso surge de la necesidad de atribuir una solución coordinada a nivel internacional a problemas determinados. Desde este punto de vista, la exigencia por parte del Consejo de Seguridad de concentrar mayores competencias no es más que otro aspecto del mismo fenómeno, aunque al mismo tiempo representa la posibilidad de empezar a controlar y delimitar un crecimiento desordenado o incluso contradictorio de regímenes especiales de derecho internacional. Una concentración, ya sea por medio del Consejo de Seguridad o de la creación de mecanismos de solución de conflictos de interpretación y competencias, puede probarse necesaria en ciertos casos y peligrosa en otros.

\section{Proyectos actuales de la CDI}

El estado que guardan algunos proyectos actuales de la $\mathrm{CDI}^{65}$ confir-

62 Zimmermann y Eberling, op. cit., nota 59, pp. 73 y ss.

63 Nolte, Georg, "Lawmaking through the Security Council", op. cit., nota 59, pp. 239 y ss.

64 Nolte, Georg, "The International Legal System: Is its Nature Changing”, Austrian Review of International and European Law, núm. 8, 2005, pp. 81-90.

65 Los documentos que a continuación se citan se encuentran en: http://wwww. un.org/law/ilc/sessions/57/57sess.htm. 
ma la impresión de un panorama mixto. Por un lado, ya no hay grandes proyectos del calibre de las convenciones sobre el derecho del mar, de tratados o de relaciones diplomáticas, como los había en fases anteriores de crecimiento del derecho de gentes. Y los proyectos ambiciosos que figuran en la agenda de la CDI, como aquél sobre los actos unilaterales de los Estados ${ }^{66}$ no parecen tener muchas expectativas. Por otro lado, algunos proyectos que conciernen preguntas clásicas y sumamente importantes, como la protección diplomática ${ }^{67}$ y las reservas a los tratados, ${ }^{68}$ registran avances importantes. En torno a la cuestión cada día más relevante de la responsabilidad de las organizaciones internacionales, es posible que la CDI se acerque a una solución desarrollada en continuidad con los artículos sobre la responsabilidad internacional del Estado, pero al mismo tiempo con independencia de éstos. ${ }^{69}$ Asimismo, hay buenas esperanzas respecto a la conclusión del tema de los recursos naturales compartidos, específicamente el de las aguas subterráneas transfronterizas. $^{70}$ En el futuro, la CDI también tendrá que enfrentarse al problema que representa el desarrollo progresivo del derecho codificado.

\section{BALANCE}

Quien habla de "la" crisis "del" derecho internacional, tiene que decir a qué se refiere exactamente, pues en la actualidad coexisten varias crisis. Todas ellas dependen, en mayor o menor medida, de problemas específicos de política internacional y de una constelación fundamental. Tal situación no es nueva, toda vez que también en épocas anteriores se

66 Eighth Report on Unilateral Acts of States by Víctor Rodríguez Cerdeño, Special Rapporteur, UN Doc. A/CN.4/557, 26.5.2005.

67 Sixth Report on Diplomatic Protection by John Dugard, Special Rapporteur, UN Doc. A/CN.4/546, 11.8.2005.

68 Tenth Report on Reservations to Treaties by Alain Pellet, Special Rapporteur, UN Doc. A/CN.4/558, 1.6.2005.

69 Third Report on Responsibility of International Organizations by Giorgio Gaja, Special Rapporteur, UN Doc. A/CN.4/553, 13.5.2005; así como: Responsibility of International Organizations-Titles and Texts of the Draft Articles Adopted by the Drafting Committee on 27.5.2005, UN Doc. A/CN.4/L.666/Rev.1, 1.6.2005.

70 Third Report on Shared Natural Resources: Transboundary Groundwaters, by Chusei Yamada, Special Rapporteur, UN Doc. A/CN.4/551, 11.2.2005; así como: UN Doc. A/CN.4/551/Add.1, 9.3.2005; Working Group on Shared Natural Resources (Groundwater)-Report of the Working Group, UN Doc. A/CN.4/L.681, 28.7.2005. 
sucedieron crecimiento, ambivalencia y crisis. Tras el gran paso que significó la Carta de las Naciones Unidas, el derecho de gentes sólo pudo avanzar lentamente. Aún así, no sería justo equiparar los esfuerzos que se han hecho en torno al desarrollo del derecho internacional con una tarea de Sísifo. Las grandes codificaciones desarrolladas durante la primera fase de la Guerra Fría colocaron fundamentos que a la fecha siguen vigentes y en práctica. Incluso los proyectos que en su momento se vieron paralizados, fueron retomados tras la descolonización o después de la caída de la Unión Soviética, y han sido llevados, al menos parcialmente, a buen término, formando hoy parte del acervo de un derecho indisputable, o bien, de un derecho que evoluciona en esa dirección.

Desde esa perspectiva, el desarrollo del derecho internacional es una historia de progreso en la cual el derecho tradicional, elaborado por conductos diplomáticos y basado en la reciprocidad, no se opone a formas más modernas de creación normativa en las que intervienen otros actores, y en donde los intereses comunitarios juegan un papel destacado. Por supuesto que en ocasiones se presenta la necesidad de fusionar viejas y nuevas concepciones del derecho internacional, como en el caso de los derechos humanos y la inmunidad del Estado. ${ }^{71} \mathrm{Y}$ por supuesto que algunos proyectos se han mostrado incoherentes, irrealizables o, en el mejor de los casos, poco exitosos. Pero en términos generales, el proyecto de antaño, consistente en desarrollar progresivamente el derecho internacional, ha logrado realizarse en muy buena medida, y al mismo tiempo, preservar una actualidad sorprendente. Lo mismo vale para las Naciones Unidas, quienes en más de un momento histórico han parecido "irrelevantes", para constatarse en el siguiente como el centro en el que convergen y se cristalizan todos los esfuerzos tendentes al reacercamiento.

Lo anterior no significa que vivamos en "el mejor de los mundos posibles", toda vez que persisten preguntas fundamentales. Con el incesante avance de la globalización, en sus más diversas manifestaciones, aumentan también los desafíos para la creación y aplicación normativas. Las fuentes e instituciones tradicionales del derecho internacional corren el peligro de no poder hacer frente a dichos riesgos. Los estándares internacionales se enfrentan a nuevas exigencias de calidad, fuerza vinculatoria,

71 Al respecto, confróntese la sentencia de la Corte Europea de Derechos Humanos en el caso Al-Adsani vs. Reino Unido, del 21.11.2001, EuGRZ, núm. 29, 15-17-2002, pp. 411-415. 
posibilidades de cambio e instrumentación, ${ }^{72}$ pudiendo ocasionar disputas políticas cada vez más intensas. Con el fin de limitar al máximo dichas disputas, algunos Estados dispuestos a tomar la inciativa internacional - en el momento, sobre todo Estados Unidos de América y sus aliados - buscarán, siempre que les sea posible, sus metas por rutas indirectas. Ello podría ocurrir con la ayuda de las coalitions of the willing o de otros estándares informales autoimpuestos, cuyo cumplimiento se intenta garantizar mediante incentivos y sanciones económicas. Tal comportamiento no está prohibido por el derecho internacional, pero puede tener considerables costes políticos y legitimatorios.

Por su parte, la idea de un renacimiento de instrumentos clásicos y formales del derecho de gentes no es nada descabellada. Así, por ejemplo, si se llegasen a confirmar las expectativas respecto al surgimiento de bloques políticos opuestos a Estados Unidos de América, como China y/o India. El rol de la Unión Europea y sus Estados miembros también sería de gran importancia para el desarrollo del derecho y el futuro carácter de las relaciones internacionales. Y las Naciones Unidas seguramente desempeñarían en este escenario, de una u otra manera, un papel central e indispensable.

72 Al respecto, véase Wolfrum, Rüdiger y Röben, Volker (eds.), op. cit., nota 59, 2005 . 